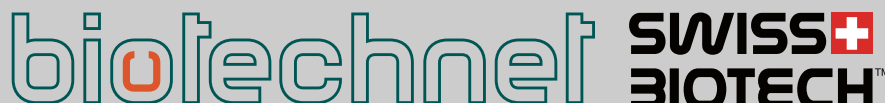

biotechnet Switzerland

Hot from the press!

\section{Quo Vadis Point-of-Care Diagnostics? Report II of the SWISS SYMPOSIUM in Point-of- Care Diagnostics 2017}

\section{Marc E. Pfeifer ${ }^{\star}$}

${ }^{*}$ Correspondence: Prof. M. E. Pfeifer, Head of the Diagnostic Systems Research Group, E-mail: marc.pfeifer@hevs.ch, Institute of Life Technologies, School of Engineering, Vice-Coordinator of the Health Technology Innovation Center (HTIC), HES-SO Valais-Wallis, Route du Rawil 47, Postfach 2134, CH-1950 Sitten 2

Abstract: U.S. studies show that the global point-of-care (POC) diagnostics market will reach $\$ 40.5$ bn by 2022 , with a compound annual growth rate (CAGR) of $10 \% .{ }^{[1]}$ This is one of the reasons why HES-SO Valais-Wallis and CSEM, acting on behalf of the NTN Swiss Biotech thematic platform in vitro Diagnostics (TP IVD), invited interested parties on October 26, 2017 to the SWISS SYMPOSIUM in Point-of-Care Diagnostics (see CHIMIA No. 12/2017). ${ }^{[2]}$ We now bring the second report on the future prospects of POC diagnostics.

Keywords: Analytical chemistry · Biomarker tracking 'Connected age' · Cost of development · Systems approach

Innovative technologies are taking giant steps forward as biosensors, lab-on-a-chip approaches, smartphones and wearable devices together with new connectivity and communication possibilities push ahead the POC diagnostics market. Driving forces are the short turnaround times, health and/or cost benefits (already proven in some cases), and the potential for allowing patients to take their health into their own hands. The spectrum of applications ranges from infectious disease detection and determination of antibiotic resistances in bacteria to autoimmune diseases and cancer.

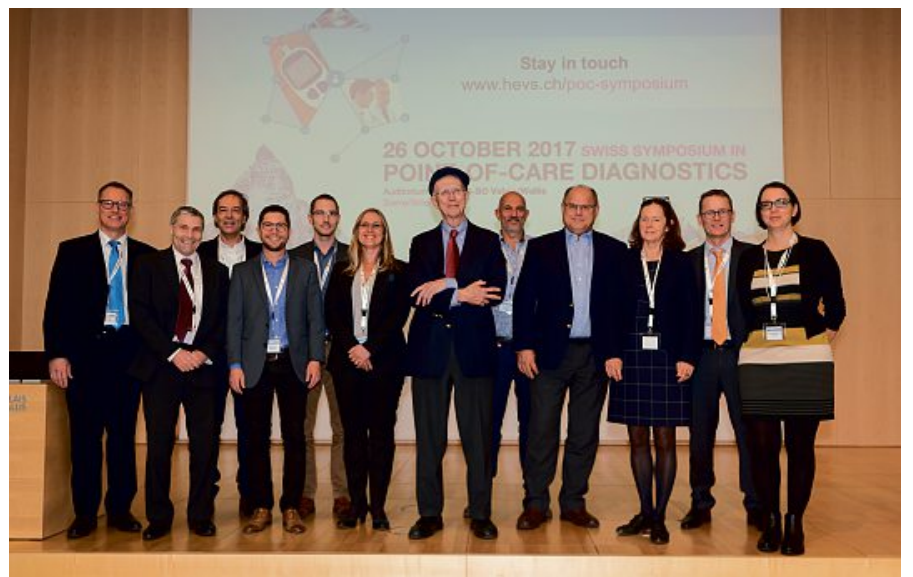

Group photo with speakers and chairmen (left to right: Prof. M. Pfeifer, HES-SO Valais, Chairman; Dr. J. Weber, BÜHLMANN Laboratories; Prof. Ph. Renaud, EPFL; Prof. J.-M. Segura, HES-SO Valais; Dr. F. Rebeaud, Abionic; Dr. M. Fleury-Siegenthaler, FOPH; Prof. G. Whitesides, Harvard University; Prof. J.-C. Sanchez, University of Geneva; Dr. P. Allibert, GenePOC; Fr. F. Amiet, Inselspital; Dr. D. Ulrich, Co-Chairman, CSEM; and Dr. S. Paoletti, CSEM). Missing on the picture: Prof. N. Senn, PMU, University of Lausanne. Photo: Caline Sian/HES-SO Valais-Wallis.

\section{Point-of-Care Diagnostics on the Move}

Healthcare without POC diagnostics is unthinkable nowadays. Diagnostic tests administered outside the central laboratory and close to the patient are a well-established reality in many countries now. 'Close-to-the-patient healthcare testing' refers, for example, to biomarker measurements at the bedside, e.g. in an intensive care unit (ICU), emergency or operating room. POC diagnostic testing also encompasses technological solutions for the general practitioner's office, pharmacies and in-home patient care. Just as the actual location of the POC testing varies, so does its intended use as well as the specific requirements and constraints. Especially in the well-developed industrial nations, aspects such as the cost-effectiveness and clinical utility of POC diagnostic devices are important factors to be considered before they can be launched onto the market. Concerns about the true benefits to patient care as well as quality/reliability issues of new POC diagnostic technologies have impeded market growth in some countries and market segments. However, the promise of short analytical turnaround times, increased mobility and server/ cloud connectivity are powerful driving forces for the development and commercialization of new POC diagnostic devices. In the past, it was thought that HIV self-testing and molecular diagnostics at the point of care would never become possible and that only technically trained staff would be able to do the analysis. It was argued, for instance, that the risk of contamination and the likelihood of creating false results would be too high.

\section{Quantified Self- and Nano-Sensors?}

What does the (longer-term) future of POC diagnostics hold? With the population aging, chronic diseases are on the rise. An affluent and well-educated population wants to be proactive in tracking and quantifying their well-being. Who does not yet have family members and friends whose wrists are adorned, for instance, with Fitbit ${ }^{\circledR}$ trackers? Will a future-generation product be able to measure other, more meaningful, parameters than the heart rate and the number of steps walked? If yes, that could signify a quantum leap in telemedicine and preventive medicine.

Healthcare can be seen as an extension of general wellness and fitness. In the United States, companies are selling test kits for at-home blood testing to monitor a variety of biomarkers including genetic and microbiome. Will the direct-to-consumer lab test market continue to grow as it did the last 5-7 years? Maybe one day our home medicine chests will comprise paper-based diagnostic tests as conceived by the Whitesides Research Group and other such groups (see separate box) along with other essential medical supplies and a thermometer. One day we will see whether this makes sense from a health economics point of view or whether it creates unnecessary anxiety and administrative costs. Implantable, electronic, tattoo-like sensors and wearables will continue to evolve, making it possible to analyse in vivo biomarkers not yet measurable due, for instance, to sensor-to-human tissue interface problems or sensitivity, reliability and other performance issues. Maybe implantable or wearable sensors are just not the right technical approach. Alternatively, nano-sensors - once intravenously injected - will travel our blood circulation 
and tissue on the lookout for cells that are becoming cancerous. Are these dreams of the future?

\section{Technical Hurdles to be Overcome}

Over the past decades, in vitro diagnostics (IVD) has seen an industrialization process: The central laboratories have become fully automated, with high-throughput instruments occupying the halls of hospital and private laboratories. Together with sophisticated sample storage facilities and data management systems, they have contributed to an enormous increase in testing efficiency. It is an incredible success story, and replacement by POC testing is almost unimaginable, yet these modern medical diagnostic facilities likely only represent an intermediate stage in the technological evolution of bioanalytical testing. As was pointed out by Prof. Philippe Renaud at the Swiss Symposium in Point-of-Care Diagnostics at HES-SO Valais in October 2017, "the smart use of simple technologies" is often a good recipe for successful product development. [2] These high-throughput instruments are based on robust engineering work with careful incremental improvements to key components of the system. This is how clinical performance requirements and reliability expectations became attainable.

However, high-sample throughput is not a requirement for a POC diagnostic device. Ideally, a broad test menu, a short turnaround time, minimally invasive sample collection and reliable measurement results are what matters. Benchtop analyzers in hospitals, pharmacies and at some general practitioners' offices fulfil these criteria more or less, but for an envisioned wearable or smartphone-like health tracking device this is extremely challenging. Simultaneous - i.e. multiplexed and accurate - quantification of several relevant biomarkers in very different concentration ranges with such a mini-laboratory device is still fiction. The accurate measurement of analytes in complex matrices such as blood or urine at nanomolar concentrations poses great challenges. Instead of parallel multi-analyte measurements, dedicated, target molecule-specific cartridges may be a workaround for the sample preparation part, followed by a more generic detection method. Microstructured polymers or paper, as used in commonly known lateral flow assays (LFA), may in some cases be sufficient for sample preparation, but for other analytes a more elaborate method may be required.

Integration or even consolidation of multiple processing and detection steps - without jeopardizing assay performance - can be an extremely complex undertaking. The development of POC diagnostic devices also comprises the conception of smart consumable vessels or cartridges to accommodate sample preparation and assay steps. For instance, the core parts of both the revogene ${ }^{\mathrm{TM}}$ real-time PCR system and the nanotechnology-based abioSCOPE ${ }^{\circledR}$ platform described by Dr Patrice Allibert and Dr Fabien Rebeaud, respectively, are the critical elements of the products where liquids are processed and detected in a certain way. ${ }^{[2]}$

\section{The Financial Bottleneck}

The cost of goods (COG) as well as the cost of development and manufacturing must also be carefully looked at when developing a new POC diagnostic device. In fact, the specific design of the consumable cartridge may significantly affect test performance as well as costs. Not surprisingly, the conception of such cartridges may absorb considerable development time. Thus Prof. George Whitesides, in his interview at the Sierre symposium, summarized the situation for the next generation of scientists and engineers as follows: "What the students need to realize is that it's a systems problem." By using the word 'system', Prof. Whitesides emphasized the fact that developing a new POC diagnostic product is an interdisciplinary endeavor. You have to think across disciplines and understand how the biological options and the design of hardware, reagents and software as well as economic aspects have to work together. Prof. Whitesides sums up: "The real problem is paying for the development. So it's ultimately an area that's limited by cost rather than by technology." A successful technology cannot have a positive impact on healthcare and society in general if it has not been moved forward towards becoming a product due to lack of finance. Having said that, the 'product development partnerships' program of the Foundation for Innovative New Diagnostics (FIND) ${ }^{[3]}$ enables the development and implementation of diagnostic solutions for low- and middle-income countries.

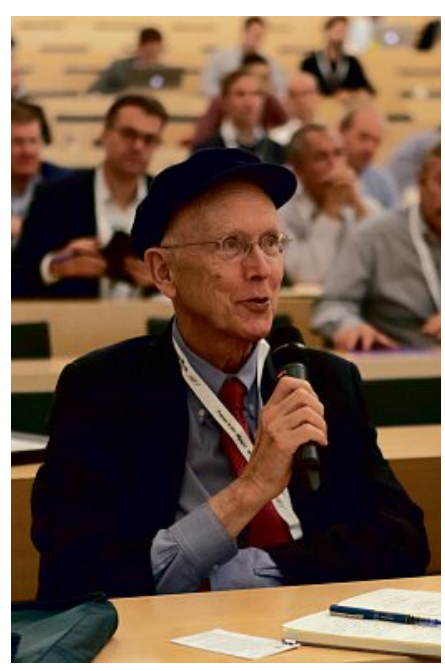

Keynote speaker Prof. George M. Whitesides during a $Q \& A$ session at the Swiss Symposium in POC Diagnostics. Photo: Caline Sian/HES-SO Valais-Wallis.

Please do not miss the opportunity to participate in the second edition of the SWISS SYMPOSIUM in Point-of-Care Diagnostics, due to take place in Fall 2018. This event will bring together representatives from research institutions, business partners and the medical community, and promote the establishment of highly promising alliances to collaborate on the future generation of POC diagnostics products.

Received: January 11, 2018

[1] https://www.zionmarketresearch.com/news/point-of-care-diagnostics-market

[2] E. Heinzelmann, Chimia 2017, 71, 872

[3] https://www.finddx.org

[4] M. S. Verma, M.-N. Tsaloglou, T. Sisky, D. Christodouleas, A. Chen, J. Milette, G. Whitesides, Biosens. Bioelectron. 2018, 99, 77.

[5] http://www.dfa.org 


\section{George M. Whitesides, Harvard University - 'Accessible Bioanalysis for the Developing World and the Point} of Care'

Modern medical infrastructures do not exist or are rare in rural and remote areas of resource-poor as well as developing countries. Concurrently, diseases such HIV, TB, malaria, Ebola, Lassa, dengue, Zika etc. are widespread in certain regions of the world or suddenly emerge to become a serious regional if not international problem. Thus, inexpensive, rapidly deployable and easy-to-use POC diagnostic devices could be of significant benefit to African, Asian and BRICS countries by providing efficient means to screen for a variety of diseases. Prof. George M. Whitesides and his team at Harvard University have developed low-cost microfluidic paper-based analytical devices ( $\mu$ PADs) with that goal in mind. More recently, Whitesides described the development of a device for a resource-limited setting that can run an enzyme-linked immunosorbent assay (ELISA) on paper for the detection of C-reactive protein (CRP), a biomarker for sepsis and inflammatory bowel disease (IBD), at a cost per kit of $\$ 0.50$ when produced on a large scale. ${ }^{[4]}$

George M. Whitesides was born on 3 August 1939. He received his PhD in chemistry from the California Institute of Technology in 1964. Prof. Whitesides has contributed to a wide range of topics including NMR spectroscopy, organometallic chemistry, molecular self-assembly, materials science, microfabrication and nanotechnology. He was awarded the Paracelsus Price of the Swiss Chemical Society in 2004 and received the Priestley Medal for a lifetime of achievement in chemistry in 2007. Today, he is interested in the development of low-cost diagnostic devices for applications in the developing world. Professor Whitesides is co-founder of the non-profit organization Diagnostics for All. ${ }^{[5]}$ Please see his keynote speech given at HES-SO Valais-Wallis on October 26, 2017:

https://portal.klewel.com/watch/webcast/swiss-poc-symposium-2017/talk/13 